\title{
Topological Tradeoffs in Autocatalytic Metabolic Pathways
}

\author{
Gentian Buzi and John Doyle
}

\begin{abstract}
Metabolic pathways in cells convert external food and resources into useful cell components and energy. In many cases the cell employs product inhibition to regulate and control these pathways. We investigate the performance of such regulation and control on certain autocatalytic pathways. Specifically, we examine how well the pathways can maintain the desired output concentrations in the presence of disturbances, such as perturbations in resources, enzyme concentrations and product demand. Using control theoretic tools, we show the effects of the pathway size, the reversibility of the intermediate reactions and the coupling of pathways through the consumption of intermediate metabolites on performance. In addition, we establish some necessary conditions on the existence of fixed points and their stability for such pathways.
\end{abstract}

\section{INTRODUCTION}

Cell metabolism converts external food and resources to useful cell components and energy, using a series of enzymatically catalyzed chemical reactions organized in metabolic pathways. One of the ways that cells regulate (control) these pathways is by product inhibition, a process through which a product of the pathway binds to an enzyme causing the enzyme to change shape and become less effective (allosteric regulation). Some of these metabolic pathways are autocatalytic, i.e., they contain reactions that consume one of the pathway's own products. Autocatalytic processes are found at every scale in biology, ecology, and technology. The most basic and also the most studied is glycolysis, which is at the heart of metabolism. The glycolysis pathway, which is the main source of anaerobic energy production, initially requires the consumption of 2 ATP molecules (energy carriers) and later in the pathway produces 4 ATP molecules [1]. In addition, metabolic pathways within the cell do not act in isolation, but are an integral part of the whole cell activity. They are coupled with other networks through the sharing of components and the exchange of their intermediate products. Glycolysis, for example, provides many of the necessary intermediates used to produce amino acids, lipids, nucleotides, and other organic molecules essential to the function of the cell [1]. In this paper we investigate pathways with the topology shown in figure 1. Specifically, these pathways are composed of a chain of reversible reactions that convert one metabolite to another and require the consumption of the final product to convert their input into the first metabolite. Additionally, the product of the pathway inhibits the enzyme that catalyzes the autocatalytic reaction and the pathway product and intermediate metabolites are consumed by other processes in the cell.

The authors are with the Control and Dynamical Systems, California Institute of Technology, Pasadena, CA, 91125, USA genti, doyledcds.caltech.edu

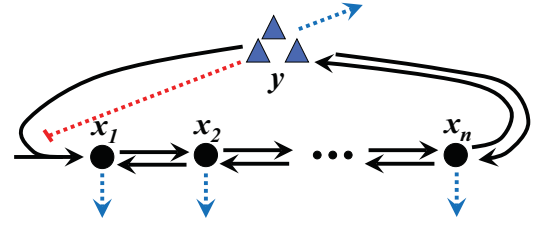

Fig. 1. The autocatalytic network is composed of a chain of reversible reactions (black arrows) that convert one metabolite (black circles) to another The pathway requires the consumption of the final product (blue triangles) to convert its input into the first metabolite. Additionally, the product of the pathway inhibits (dotted red line) the enzyme that catalyzes the autocatalytic reaction and the pathway product and intermediate metabolites are consumed by other processes in the cell (dotted blue arrows).

Nonlinear stability and region of attraction properties for such autocatalytic pathways (with application to glycolysis pathway) are the subject of [2], [3], focusing on the case in which the intermediate reactions are not reversible and there is no intermediate metabolite consumption. For general biochemical networks, stability and the existence of steady states have been the subject of study for many decades. Results regarding the number of steady states and convergence properties around steady states for rather general networks with mass-action kinetics are established in [4], [5], [6]. Local and global convergence properties have been established for many network topologies such as monotone dynamical systems [7], [8] and cyclic interconnection networks [9], [10], [11], [12].

Here, we are primarily interested on the performance of autocatalytic metabolic pathways near their biological operating point. In particular, we study how the pathway can best maintain the desired output concentration in the presence of disturbances such as perturbations in resource availability, product demand and enzyme concentrations using feedback inhibition. Using tools from linear systems theory, our previous work on a simple two-state pathway (figure $2 \mathrm{a}$ ) showed that autocatalysis can aggravate controller performance [13]. We have shown that this reduced model captures the essence of glycolysis pathway and glycolytic oscillations, but it is sensitive to parameter perturbations and exhibits a very rich dynamic behavior. Perturbations in the parameters can make the system undergo a variety of bifurcations, such as saddle-node, Hopf and homoclinic bifurcations [2], [14]. This can cause the system to oscillate (limit cycles) or even crash, implying that none of the theorems or existing theory that rule out such behavior are applicable. Since this level of parameter sensitivity of the dynamics is not immediately obvious from the structure of the reduced model or the underlying biology, it is important to explore more realistic pathway models and examine how perturbations in the structure of the chemical reactions in the pathway model 


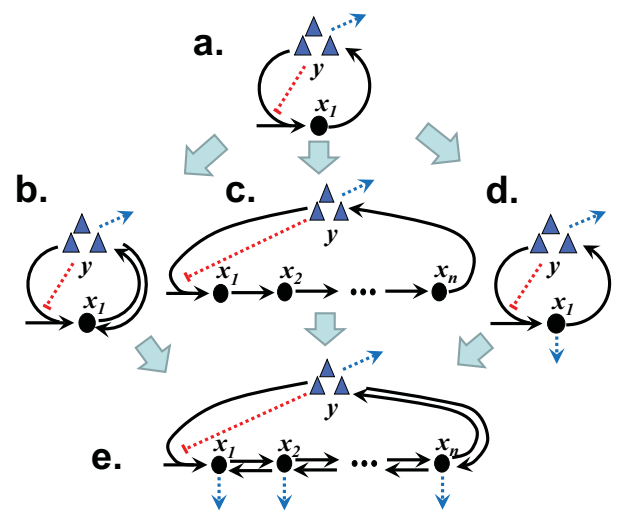

Fig. 2. Performance in the different network topologies (reversibility of intermediate reactions (b), large pathways (c) and intermediate metabolite consumption (d)), is compared to that of the simplest two-state pathway (a).

effect dynamics and performance. In this paper, we investigate how the size of the pathway, the reversibility of the intermediate reactions and the coupling of pathways through the consumption of intermediate metabolites effect their performance (figure 2). In the case when there is coupling to other pathways via intermediate metabolite consumption, we establish some necessary conditions on the existence of fixed points.

The rest of the paper is organized as follows: We introduce the pathway model in the next section, followed by a discussion of control and performance in section II-A. Next we consider the different topologies separately, by looking at large pathways in section III, pathways with reversible reactions in section IV and pathways with intermediate metabolite consumption in section $\mathrm{V}$. The more general pathways are discussed in section VI.

\section{MODEL DESCRIPTION}

Consider the autocatalytic metabolic pathway with multiple intermediate metabolite reactions (figure 1)

$$
\begin{aligned}
& u+a y \rightarrow^{f} x_{1}
\end{aligned}
$$

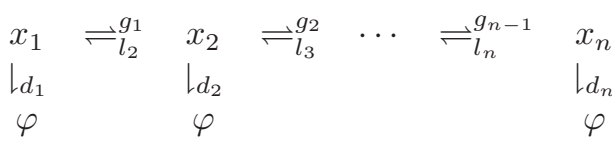

$$
\begin{aligned}
& x_{n} \rightleftharpoons l_{l_{y}}^{g_{n}}(a+b) y \longrightarrow^{g_{y}} \varphi
\end{aligned}
$$

Here, $u$ is some precursor and source of energy for the pathway with no dynamics associated, $y$ denotes the product of the pathway, $x_{i}$ are intermediate metabolites, $\varphi$ is a null state, $a$ is the number of $y$ molecules that are invested in the pathway, and $a+b$ is the number of $y$ molecules produced. $A \rightarrow{ }^{f} B$ denotes a chemical reaction that converts chemical species $A$ to species $B$ at rate $f$ and $A \rightleftharpoons B$ denotes a reversible reaction (i.e., both the reaction that converts $A$ to $B$ and the one that converts $B$ to $A$ are present). The

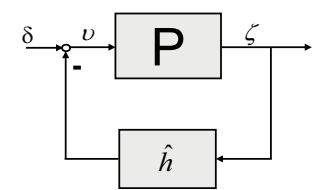

Fig. 3. Block diagram of system (2).

corresponding ordinary differential equations are

$$
\begin{aligned}
\dot{x}_{1}= & f(y)+l_{2}\left(x_{2}\right)-g_{1}\left(x_{1}\right)-d_{1}\left(x_{1}\right) \\
\dot{x}_{2}= & g_{1}\left(x_{1}\right)+l_{3}\left(x_{3}\right)-g_{2}\left(x_{2}\right)-l_{2}\left(x_{2}\right)-d_{2}\left(x_{2}\right) \\
& \vdots \\
\dot{x}_{n-1}= & g_{n-2}\left(x_{n-2}\right)+l_{n}\left(x_{n}\right)-g_{n-1}\left(x_{n-1}\right) \\
& -l_{n-1}\left(x_{n-1}\right)-d_{n}\left(x_{n-1}\right) \\
\dot{x}_{n}= & g_{n-1}\left(x_{n-1}\right)+l_{y}(y)-g_{n}\left(x_{n}\right) \\
& -l_{n}\left(x_{n}\right)-d_{n}\left(x_{n}\right) \\
\dot{y}= & (a+b) g_{n}\left(x_{n}\right)-a f(y)-(a+b) l_{y}(y)-g_{y}(y),
\end{aligned}
$$

for $x_{i} \geq 0, y \geq 0$. Here, $g_{1}, \ldots, g_{n}, l_{2}, \ldots, l_{n}, l_{y}, d_{1}, \ldots, d_{n}$, and $g_{y}$ are continuous, monotone, increasing functions that vanish at 0 . These assumptions are consistent with large classes of chemical kinetics models such as mass-action and Michaelis-Menten. The rate $f(y)$ of the autocatalytic reaction captures the negative feedback of the output $(y)$ via the inhibition of the catalyzing enzyme of the first reaction. We choose $f(y)=\frac{V y^{q}}{1+\gamma y^{h}}$ that is consistent with biological intuition and experimental data (in the case of the glycolysis pathway [15], [16]). In this parameterization $V>0$ depends on the concentration of the input $(u), q>0$ captures the strength of autocatalysis and $\gamma>0$ and $h>0$ capture the strength of inhibition (negative feedback). Note that $f$ is not monotone and captures the coupling between the autocatalysis and inhibition. For the rest of the paper, we take $a=b=1$ and note that the generalization of the results for $a>0$ and $b>0$ is straightforward.

\section{A. Feedback Mechanism, Sensitivity Function, Zeros}

If a nonzero fixed point $(\bar{x}, \bar{y}) \succ(0,0)$ of (1) exists, then without loss of generality we set $\bar{y}=1$ (and assume so for the rest of the paper). Define $\hat{h}:=\frac{\gamma}{\gamma+1} h$, and

$$
\begin{array}{ll}
V_{0}:=f(1)=\frac{V}{1+\gamma} & r_{i}:=\left.\frac{\partial}{\partial x_{i}} l_{i}\left(x_{i}\right)\right|_{\bar{x}} \\
k_{i}:=\left.\frac{\partial}{\partial x_{i}} g_{i}\left(x_{i}\right)\right|_{\bar{x}} & \eta_{i}:=\left.\frac{\partial}{\partial x_{i}} d_{i}\left(x_{i}\right)\right|_{\bar{x}} \\
k_{y}:=\left.\frac{\partial}{\partial y} g_{y}\left(y_{i}\right)\right|_{1} & r_{y}:=\left.\frac{\partial}{\partial y} l_{y}(y)\right|_{1} .
\end{array}
$$

The dynamics of (1) near the fixed point are given by the following linear system

$$
\left[\begin{array}{c}
\dot{x} \\
\dot{y}
\end{array}\right]=\left(J_{0}-\hat{h} B C\right)\left[\begin{array}{l}
x \\
y
\end{array}\right]
$$


for $x \in \mathbb{R}^{n}, y \in \mathbb{R}$ and where $B:=V_{0}[1,0, \ldots, 0,-1]^{T}$, $C:=[0,0, \ldots, 0,1], J_{0}$ is

$\left[\begin{array}{ccccccc}-m_{1} & r_{2} & 0 & \cdots & 0 & 0 & q V_{0} \\ k_{1} & -m_{2} & r_{3} & & 0 & 0 & 0 \\ 0 & k_{2} & -m_{3} & \ddots & 0 & 0 & 0 \\ & & \ddots & \ddots & & & \vdots \\ 0 & 0 & 0 & & -m_{n-1} & r_{n} & 0 \\ 0 & 0 & 0 & & k_{n-1} & -m_{n} & r_{y} \\ 0 & 0 & 0 & \cdots & 0 & 2 k_{n} & -\delta_{y}-q V_{0}\end{array}\right]$

and for all $i, m_{i}:=k_{i}+r_{i}+\eta_{i}, r_{1}:=0$ and $\delta_{y}=k_{y}+2 r_{y}$. Notice that equation (2) can be viewed as the closed-loop dynamics of

$$
\begin{aligned}
& \dot{\xi}=J_{0} \xi+B \nu \\
& \zeta=C \xi .
\end{aligned}
$$

using a proportional (negative) feedback controller of gain $\hat{h}$ (i.e., $\nu=-\hat{h} \zeta$ ). Biologically, the gain $\hat{h}$ captures the strength of the inhibition of the autocatalytic reaction by the pathway product (in the case of glycolysis, it captures the strength of inhibition of the catalyzing enzyme PFK by ATP [2]). Figure 3 shows the feedback structure of system (3), where $P(s):=C\left(s I-J_{0}\right)^{-1} B$. In this paper, we explore the performance of the controller on the different network topologies shown in figure 2 by investigation of the sensitivity function $S$, given by $S(s)=\frac{1}{1+\hat{h} P(s)}$. $S$ captures the ability of the system to reject many relevant disturbances, such as perturbations $\delta$ in the pathway product consumption rate (i.e., perturbations of the RHS of equation (2) by $[0, \ldots, 0,1]^{T} \delta$ ) or in the consumption rate of other metabolites. In the topologies we investigate, the plants $P$ have right half-plane (RHP) zeros which introduce theoretical limits on performance captured by a special form of the Bode's integral formula [13],

$$
\frac{1}{\pi} \int_{0}^{\infty} \ln |S(j \omega)| \frac{2 z}{z^{2}+\omega^{2}} d \omega=M \geq 0
$$

where $z$ is a RHP zero and $M=\ln \left|\frac{z+p}{z-\bar{p}}\right|>0$ if the openloop plant has a RHP pole $p$, and $M=0$ otherwise. Notice that these limits are exacerbated by smaller magnitude RHP zeros, since it severely penalizes performance in frequencies below $z$. Reference [13] characterizes the limits and the tradeoffs involved for a special form of the two-state model shown in figure 2a. In particular it shows that performance improves as the concentration of the intermediate enzyme increases (higher intermediate reaction rate). Here, using the two-state model in figure $2 \mathrm{a}$ as a basis for comparison, we investigate how limits on performance change as this two-state model is extended to include multiple intermediate states (figure 2c), reversibility of the intermediate reactions (figure $2 \mathrm{~b}$ ) and consumption of intermediate metabolites by other pathways (figure $2 \mathrm{~d}$ ).

Comparison of performance in these different topologies can be tricky, since these topologies imply fundamental changes in the "plant" and the number and locations of the fixed points of the (nonlinear) closed loop system (1). The
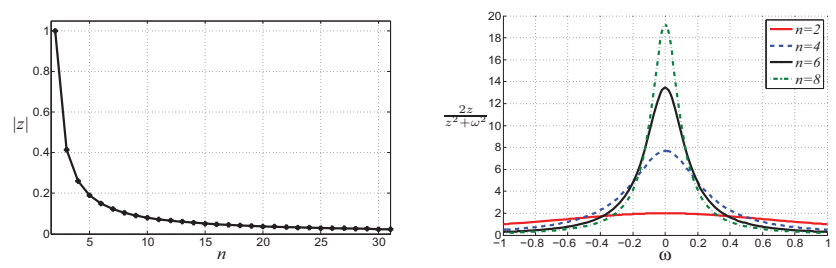

Fig. 4. (left) The magnitude of the RHP zero decreases as pathway size increases. (right) The weight in the Bode integral gets tighter around 0 for longer pathways. This means the price paid for performance at small frequencies increases substantially as the number of intermediate reactions increases.

assumption that the (operating) equilibrium concentration of the pathway output $y$ is fixed and normalized to 1 for all topologies makes such comparisons appropriate, since it directly addresses the question of which topology is easier to control, i.e., in which pathway topology the controller can best maintain the desired concentration of the output $y$ of the pathway in the presence of disturbances.

\section{LONGER PATHWAYS}

In this section we investigate the topology of figure $2 \mathrm{c}$, i.e., a pathway of size $n+1$ with no reversible reactions and no consumption of any of the intermediate metabolites (in equation $\left.(1), l_{y}(y)=0, l_{i}\left(x_{i}\right)=0, d_{i}\left(x_{i}\right)=0, \forall i\right)$. Then

$$
\begin{array}{rcc}
P(s) & = & C\left(s I-J_{0}\right)^{-1} B \\
& =V_{0} \frac{\prod_{i=1}^{n}\left(k_{i}+s\right)-2 \prod_{i=1}^{n} k_{i}}{\left(k_{y}+q V_{0}+s\right) \prod_{i=1}^{n}\left(k_{i}+s\right)-2 q V_{0} \prod_{i=1}^{n} k_{i}} .
\end{array}
$$

The coefficients of the numerator of $P(s)$, given by

$$
\begin{aligned}
n_{1}(s) & =\prod_{i=1}^{n}\left(k_{i}+s\right)-2 \prod_{i=1}^{n} k_{i} \\
& =s^{n}+\left(k_{1}+\cdots+k_{n}\right) s^{n-1}+\cdots+\left(-\prod_{i=1}^{n} k_{i}\right),
\end{aligned}
$$

do not have the same sign. This implies that the polynomial $n_{1}(s)$ is not Hurwitz stable and therefore $P(s)$ has at least one RHP zero and equation (4) holds for the n-D pathway.

Let us look at the case where $k_{i}=k$, for all $i$. Define $\hat{h}_{d}(n):=q+\frac{k_{y}}{V_{0}} \frac{\theta(n)}{2+\theta(n)}, \theta(n):=(\sec (\pi /(n+1)))^{n+1}$, then the system is stable for gains $q-1<\hat{h}<\hat{h}_{d}(n)$ [3], [11]. This implies that, in general, as the pathway size increases, the upper bound on stable feedback gains $\hat{h}$ decreases. The zeros of $P(s)$ are given by $s=k\left(\left|2^{\frac{1}{n}}\right| e^{j \frac{2 \pi i}{n}}-1\right), \forall i$. As expected, there is at least one zero in the RHP. The magnitude of this RHP zero decreases as the size of the pathway $(n)$ increases (figure 4 left).

This fact, coupled with the conservation law given by (4), implies that as the number of intermediate reactions grows the price paid for performance at small frequencies increases substantially (figure 4 right). So the increase in the intermediate metabolite reactions has two main consequences.

- The price for good performance at low frequencies increases as the magnitude of the RHP zero gets smaller.

- The upper bound on the feedback gains gets smaller, so the range of stable gains gets smaller.

For example look at the DC gain (steady state error) of a n-D system with $q=2, k_{y}=V_{0}=1$. From (5) we get $P(0)=1$ and the DC gain $S(0)=\frac{1}{1+\hat{h}}$. It is clear that the DC gain improves as the gain increases, however the 

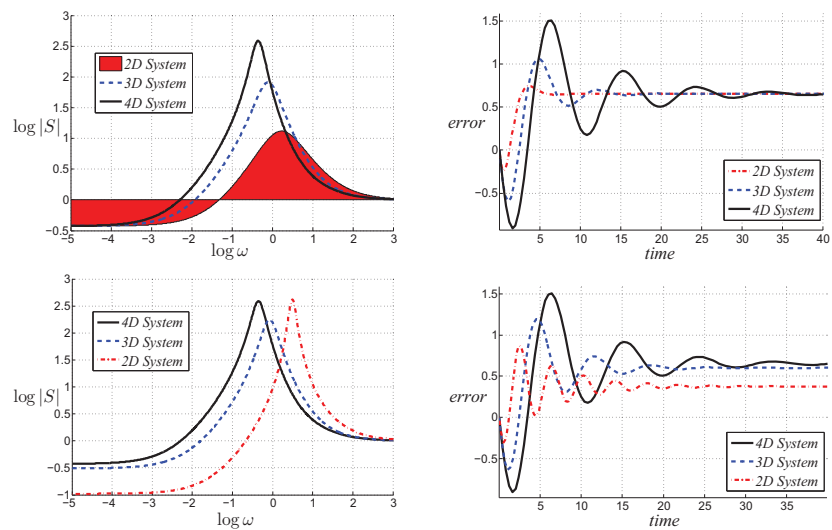

Fig. 5. The performance of two-state, three-state and four-state systems is compared. The intermediate reaction rates are $k_{1}=k_{2}=k_{3}=1$ and $q=2, k_{y}=V_{0}=1$. (top-left) Plot of $\log |S|$ for the same feedback $\hat{h}=3.8$, shows that the higher dimension systems pay a higher price at higher frequencies for similar performance at low frequencies (top-right) Closed-loop system step response shows that the same gain $\hat{h}=3.8$ yields the same steady state error for all 3 systems. However higher dimensional systems have poorer transient response. (bottom-left) Lower dimensional systems have access to higher gains, which means that they can get better performance at low frequencies. The plot of $\log |S|$ for $\hat{h}=5.5,4,3.8$ for two-state, three-state and four-state systems respectively, illustrates the difference in performance at low frequencies, and the price paid at higher frequencies (comparable for all 3 systems). (bottom-right) Closed-loop system step response shows that access to higher gains for the low dimension systems $\left(\hat{h}_{2 D}=5.5, \hat{h}_{3 D}=4, \hat{h}_{4 D}=3.8\right)$ achieves smaller steady state error and better transient response as well.

maximum $\hat{h}$ that still stabilizes the system gets smaller as $n$ increases. Therefore lower dimensional systems can achieve better DC gain (steady state error). Figure 5 illustrates the consequences of increased pathway size on performance by looking at $\log |S|$ and the step response for comparable twostate, three-state and four-state systems.

In general, long pathways in the cell can be biochemically unavoidable or even necessary (such as the intermediate metabolites are crucial to other processes in the cell). The cell uses means such as employing metabolite channeling [17] instead of free diffusion, to counteract the adverse effect of large pathway size.

\section{REVERSIBLE REACTIONS}

We investigate the topology of figure $2 b$, i.e., a pathway of size 2 with one reversible intermediate reaction, but no consumption of the intermediate metabolite (in equation (1) $\left.n=1, d_{1}\left(x_{1}\right)=0\right)$.

The system is stable for gains $q-\frac{1}{V_{0}} k_{y}<\hat{h}<q+$ $\frac{1}{V_{0}}\left(k_{1}+k_{y}+2 r_{y}\right)$ and so the presence of the reversible reactions increases the range of stable gains $\hat{h}$. This suggests that the reversible reactions make the pathway easier to control. There is still a RHP zero at $s=k_{1}$ since $P(s)=$ $-V_{0} \frac{s-k_{1}}{p_{J_{0}}(s)}$, where $p_{J_{0}}$ is the characteristic polynomial of $J_{0}$. This implies that equation (4) holds for this system. However, because the range of the stable gains is larger for higher reversible reaction (RR) rates, the same feedback gain is more "robust". This suggests that systems with higher RR rates have better performance (i.e., smaller peak in the $\log |S|$, and smaller oscillations in the step response, (figure 6 top-left, top-right). It also means that higher gains can
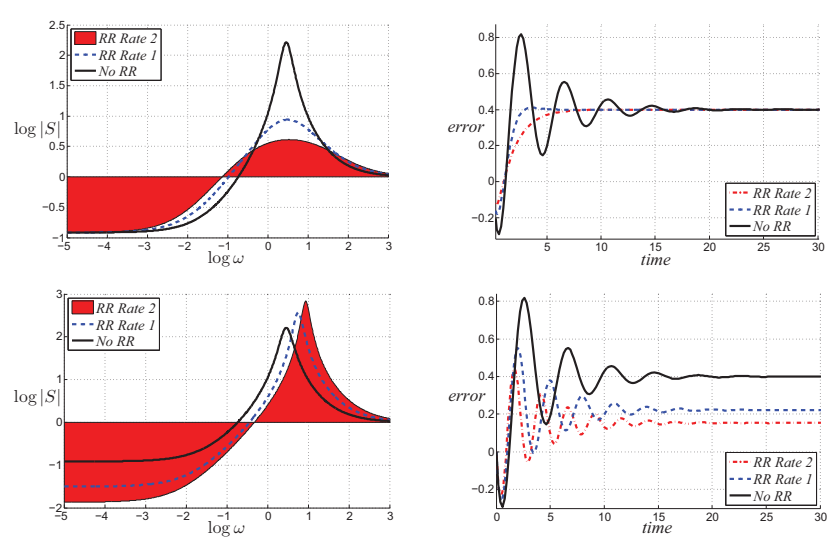

Fig. 6. The performance of three two-state models with $k_{1}=1, q=$ $2, k_{y}=1, V_{0}=1$ and $r_{y}=2,1,0$ is compared. (top) Using the same feedback gain $\hat{h}=3.5$, the systems with higher reversible reaction (RR) rates have better performance (i.e., smaller peak in $\log |S|$ (top-left), and smaller oscillations in the step response (top-right)). (bottom) Models with higher reversible reaction rates allow for higher stabilizing gains. Using $\hat{h}=$ $7.5,5.5,3.5$ respectively, we obtain better performance at low frequencies (bottom-left) and better steady state error (bottom-right) for the systems with higher RR rates.

be used for systems with high RR rates to obtain better performance at low frequencies and better steady state error without a meaningful increase in performance losses at high frequencies and qualitatively similar oscillations in the step response, as shown in figure 6 bottom-left and bottom-right.

The improvement in performance by having reversible reactions is to be expected, since the reversible reactions act as local feedback loops. For example the pathway studied in this section, is (locally) equivalent to a two state pathway with no reversible reaction, but where the product $y$ controls both the autocatalytic and the intermediate reaction.

\section{CONSUMPTION OF INTERMEDiates}

For the topology of figure 2 d, i.e., a pathway of size 2 with a single intermediate metabolite and no reversible intermediate reaction, but with consumption of the intermediate metabolite from external sources (in equation (1) $\left.n=1, l_{y}(y)=0\right)$, a fixed point $\left(x_{0}, y_{0}\right) \succ(0,0)$ exists only if $d_{1}\left(x_{0}\right)<g_{1}\left(x_{0}\right)$. I.e., there cannot be more intermediate metabolite consumption by other pathways than the current pathway. If the desired operating fixed point $\left(x_{0}, 1\right)$ exists, then it is stable for

$$
q-\frac{1}{V_{0}} k_{y} \frac{k_{1}+\eta_{1}}{k_{1}-\eta_{1}}<\hat{h}<q+\frac{1}{V_{0}}\left(k_{1}+k_{y}+\eta_{1}\right)
$$

and it can be shown that it is open-loop $(\hat{h}=0)$ stable when $\eta_{1}$ is close enough to $k_{1}$. Notice that $\eta_{1}$ cannot be larger than $k_{1}$, since for $\delta:=\eta_{1}-k_{1}>0$ and $\delta<k_{y} \frac{\eta_{1}+k_{1}}{\eta_{1}+k_{1}+k_{y}}$, the system is not stabilizable.

Under the above conditions, the consumption of the intermediate increases the range of stable gains $\hat{h}$. Similar to the RR case, this suggests that the consumption of the intermediate metabolite might make the pathway easier to control. However, the plant has a RHP zero at $s=k_{1}-\eta_{1}$ since $P(s)=-V_{0} \frac{s-k_{1}+\eta_{1}}{p_{A}(s)}$. This implies that as $\eta_{1}$ increases the magnitude of the RHP zero gets smaller, thus imposing harder constraints on performance. There appears to be 

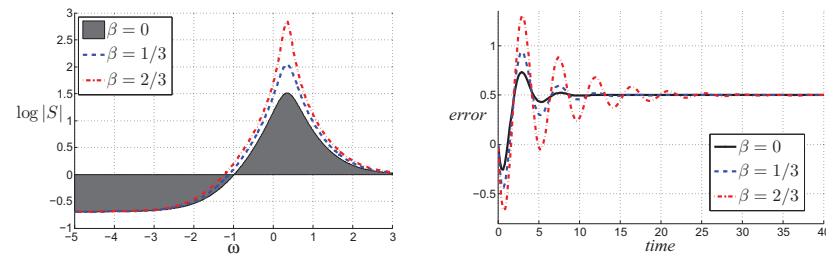

Fig. 7. Performance of three 2D models with $k_{1}=1, q=2, k_{y}=1$, and $\eta_{1}=\beta k_{1}$ with $\beta=0, \frac{1}{3}, \frac{2}{3}$ is compared. Using the same feedback gain $\hat{h}=3$, the systems with less consumption of intermediates have better performance, which corresponds to a smaller peak in $\log |S|$ plot, and less oscillations in the step response.

another tradeoff, and it is not immediately clear whether or not the consumption of intermediates helps the performance of the system. Define $m(x):=g_{x}(x)+d_{x}(x)$ and $\alpha(x)$ such that $d(x)=\alpha(x) m(x)$. This results in a system of the form;

$$
\begin{array}{ccc}
\dot{x} & = & \frac{V y^{q}}{1+\gamma y^{h}}-m(x) \\
\dot{y} & = & (2-\alpha(x)) m(x)-\frac{V y^{q}}{1+\gamma y^{h}}-g_{y}(y) .
\end{array}
$$

These equations show that consumption of intermediates has the same effect as reducing the net product of the pathway. For example, if $g_{x}(x)=k x$ and $d_{x}(x)=\beta k x$, then $\alpha=\frac{\beta}{\beta-1}$ and this pathway is equivalent to a pathway with $g_{x}(x)=(1+\beta) k x$ that produces $(2-\alpha)$ molecules of $y$ for each molecule $y$ invested. As Figure 7 shows, larger $\beta$ leads to worse tradeoffs on achievable performance and more oscillations for the same steady state error.

\section{General Pathways}

We now consider the general autocatalytic metabolic pathway given by equation (1) (figure 1).

\section{A. Existence of the Fixed Point}

Let us first consider $l_{y}=0, l_{i}=0, \forall i$, i.e., no reversible intermediate reactions. Assume that the fixed point $(\bar{x}, \bar{y}) \succ$ $(0,0)$ exists, then

$$
\left(2 \prod_{i=1}^{n} \frac{g_{i}\left(\bar{x}_{i}\right)}{g_{i}\left(\bar{x}_{i}\right)+d_{i}\left(\bar{x}_{i}\right)}-1\right) f(\bar{y})=g_{y}(\bar{y}) .
$$

This implies that the fixed point $(\bar{x}, \bar{y}) \succ(0,0)$ exists only if

$$
2 \prod_{i=1}^{n} p_{i}\left(\bar{x}_{i}\right)-1>0
$$

where $p_{i}\left(\bar{x}_{i}\right):=\frac{g_{i}\left(\bar{x}_{i}\right)}{g_{i}\left(\bar{x}_{i}\right)+d_{i}\left(\bar{x}_{i}\right)}$. We can think of $p_{i}\left(\bar{x}_{i}\right)$ as the "rate" that a molecule $x_{i}$ will continue to the next step of the pathway (i.e., will be converted into $x_{i+1}$ ), and $1-$ $p_{i}\left(\bar{x}_{i}\right)=\frac{d_{i}\left(\bar{x}_{i}\right)}{g_{i}\left(\bar{x}_{i}\right)+d_{i}\left(\bar{x}_{i}\right)}$ the rate that it will be used by another pathway. Then the rate that a molecule $x_{1}$ (produced by the first reaction through the investment of 1 molecule of $y$ ) will ultimately be converted into 2 molecules of $y$ and not be used by other pathways is given by $\prod_{i=1}^{n} p_{i}\left(\bar{x}_{i}\right)$. So for each investment of 1 molecule of $y$ at the start, the pathway returns $E_{p}[y]=2\left(\prod_{i=1}^{n} p_{i}\left(\bar{x}_{i}\right)\right)$ molecules of $y$ at the end.

Condition (6) states that for a fixed point to exist, the return $E_{p}[y]$ should exceed the value of the investment, i.e., each molecule $y$ invested must produce at least one molecule of $y\left(E_{p}[y]>1\right)$. So in order for the pathway to have a stable operating fixed point it must not allow excessive consumption of the intermediate metabolites. For example, let $d_{i}\left(x_{i}\right)=\alpha_{i} g_{i}\left(x_{i}\right)$, then condition (6) becomes

$$
\prod_{i=1}^{n} \frac{1}{1+\alpha_{i}}>\frac{1}{2} \text {. }
$$

If $\alpha_{i}=\alpha$ for all $i=1, \ldots, n$ then $\alpha<\sqrt[n]{2}-1$. So for a pathway of size $10, \alpha$ has to be less than 0.072 , i.e., only a small percentage of the intermediate metabolites can be consumed at each step.

In the general case, where the intermediate reactions are reversible, we can get a similar condition to (6) by considering the "net flow" $g_{i}\left(x_{i}\right)-l_{i+1}\left(x_{i+1}\right)$ through the pathway (i.e., define $p_{i}:=\frac{g_{i}-l_{i+1}}{g_{i}-l_{i+1}+d_{i}}, \forall i$, with $l_{n+1}=l_{y}$ ).

\section{B. Stability of the Fixed Point}

For the cases when a nonzero fixed point $(\bar{x}, 1) \succ(0,0)$ of (1) exists, we establish bounds on gains $\hat{h}$ that guarantee stability of the pathway for arbitrary size $(n)$ and arbitrary values of intermediate reaction rates $\left(g_{i}, d_{i}, l_{i}, l_{y}\right)$

Proposition 1: (a) Let $\hat{h}_{r}:=q+\frac{1}{3} \frac{k_{y}}{V_{0}}$ and $\hat{h}_{s}:=q-\frac{k_{y}}{V_{0}}$. If $\hat{h}_{s}<\hat{h} \leq \hat{h}_{r}$, then (2) is stable for arbitrary size $(n)$ and arbitrary values of $\left(k_{i}, \eta_{i}, r_{i}, r_{y}\right)$ at equilibrium.

(b) The bounds above are tight, i.e. for any gain $\hat{h} \notin$ $\left(\hat{h}_{s}, \hat{h}_{r}\right]$, there exists an unstable pathway.

Proof: (a) Proved directly by showing diagonal dominance of

$$
Q:=\left[\begin{array}{cc}
I_{n} & 0 \\
0 & 2
\end{array}\right]^{-1}\left(J_{0}-\hat{h} B C\right)\left[\begin{array}{cc}
I_{n} & 0 \\
0 & 2
\end{array}\right] .
$$

(b) For $\hat{h} \leq \hat{h}_{s}$, let $n=1, r_{y}=0, d_{1}=0$ in (2). For these parameter values, the resulting two-state system (2) is unstable.

We now show we can construct an unstable system for any $\hat{h}=\hat{h}_{r}+\epsilon, \epsilon>0$.

In equation (2), let $r_{y}=0$, and for all $i, r_{i}=0, \eta_{i}=0$, $k_{i}=\frac{2}{3} k_{y}-\epsilon V_{0}=k>0$. The characteristic polynomial $p_{J}(s)$ of $J_{0}-\hat{h} B C$ is given by

$$
\begin{aligned}
p_{J}(s)= & \left(s+k_{y}+V_{0}(q-\hat{h})\right) \prod_{i=1}^{n}\left(s+k_{i}\right) \\
& -2 V_{0}(q-\hat{h}) \prod_{i=1}^{n} k_{i} \\
= & (s+k)^{n+1}+k^{n}\left(k+3 \epsilon V_{0}\right) .
\end{aligned}
$$

The eigenvalues $\lambda_{i}$ are then given by $\lambda=k(\omega-1)$ such that $\omega^{n+1}=\left(-1-\epsilon_{1}\right)^{1 /(n+1)}$, where $\epsilon_{1}:=3 \epsilon \frac{V_{0}}{k}$. The real part of the eigenvalue $\lambda_{0}$, is given by $\Re\left(\lambda_{0}\right)=k(-1+\gamma(n+1))$, where

$\gamma(n):=\left(1+\epsilon_{1}\right)^{1 / n} \cos \frac{\pi}{n}=1+\frac{1}{n} \log \left(1+\epsilon_{1}\right)+O\left(\frac{1}{n^{2}}\right)$. This implies that for $n$ large enough, the system (2) is unstable since $\gamma(n)>1$ and therefore $\Re\left(\lambda_{0}\right)>0$.

\section{CONCLUSIONS}

Output feedback regulation of the pathway shown in figure 1, can counteract the destabilizing effects of the positive feedback generated by autocatalysis, thus guaranteeing the local stability of the operating fixed point. However the 


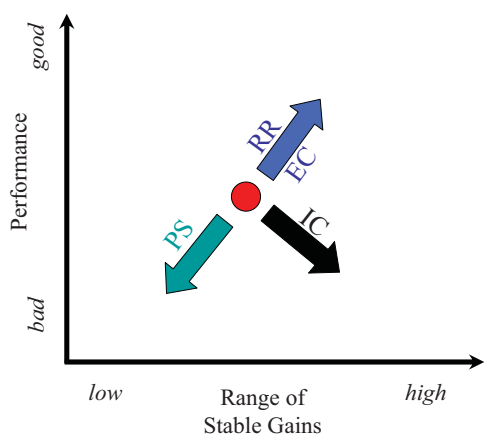

Fig. 8. The effect of increases in enzyme concentrations (EC), pathway size (PS), intermediate metabolite consumptions (IC), and reversible reactions (RR) on the performance and range of stable feedback gains.

structure of autocatalysis limits the range of stable feedback gains $\hat{h}$, because the product of the pathway both controls and is consumed by the first reaction. An upper bound $\hat{h}_{d}(n)$ on the feedback gains $\hat{h}$ suggests that the range of available stable gains gets smaller as the pathway size increases. On the other hand, consumption of intermediate metabolites and the presence of reversible reactions increases the range of stable gains. For the general model described by (1) there exist lower and upper bounds, respectively $\hat{h}_{s}, \hat{h}_{r}$, on the feedback gain $\hat{h}$ that guarantee stability for arbitrary pathway size as well as arbitrary values of both the (reversible) reaction rates and the intermediate consumption rates. These bounds are tight in the sense that for gains that lie outside the ranges established by these bounds, we can construct specific unstable pathways.

The existence of a RHP zero $z$ is at the core of many of the limitations imposed on performance for these pathways, captured by a special form of the Bode Sensitivity Integral. These limitations are aggravated by smaller magnitude RHP zeros. As shown in [13], high concentration of catalyzing enzymes leads to an increase in the rates of the intermediate reactions $\left(k_{i}\right)$, which makes the pathway more stable (i.e., there is a larger range of stable gains available) and increases the magnitude of $z$ (i.e., "softens" the hard limits on performance).

Increased pathway size has the opposite effect of increasing the enzyme concentrations on pathway performance. An increase in the size of the chain of enzymatically catalyzed intermediate reactions in the autocatalytic pathways causes two adverse effects on performance: tradeoffs on performance limits are exacerbated (as $z$ becomes smaller) and the range of available stable gains is reduced, which makes the operating gains less robust and reduces the achievable performance objectives.

Consumption of intermediates in autocatalytic metabolic pathways results in less resources available to convert to the product of the pathway, which effectively reduces the net product of the pathway (i.e., how much product $y$ is produced per unit $y$ invested in the autocatalytic step). This effective reduction in net output production makes the pathway harder to control because it corresponds with the RHP zero getting smaller, and thus aggravating the tradeoffs on performance limits. Additionally, these pathways must enforce a positive return in the energy investment, i.e., for each unit of $y$ invested at the beginning, it must produce at least that much $y$ produced by the end of the pathway. Therefore, the loss of the intermediates to the other pathways must be only a fraction of the available resources, otherwise excessive consumption of these intermediates causes the pathway to crash.

On the other hand, the presence of reversible reactions makes the autocatalytic pathways easier to control, as they function as "release valves" by making higher stable gains available, thus providing more robustness and better achievable performance objectives. Figure 8 shows a summary of the role of concentration of catalyzing enzymes, pathway size, consumption of intermediate metabolites, and reversible reactions in determining the stability and performance of the pathway.

\section{REFERENCES}

[1] B. Alberts, A. Johnson, J. Lewis, M. Raff, K. Roberts, and P. Walter, Molecular Biology of the Cell, $4^{\text {th }}$ ed. Garland, 2002.

[2] G. Buzi, U. Topcu, and J. C. Doyle, "Quantitative nonlinear analysis of autocatalytic networks with applications to glycolysis," in Proc of American Control Conference, 2010.

[3] — "Compositional analysis of autocatalytic networks in biology," in Proc of American Control Conference, 2010.

[4] M. Feinberg, "Chemical reaction network structure and the stability of complex isothermal reactors: II Multiple steady states for networks of deficiency one," Chemical Engineering Science, no. 43, pp. 1-25, 1988

[5] — " "The existence and uniqueness of steady states for a class of chemical reaction networks," Arch Rational Mech Analysis, no. 132, pp. 311-370, 1995.

[6] F. Horn and R. Jackson, "General mass action kinetics," Arch Rational Mech Analysis, vol. 47, no. 2, pp. 81-116, 1972.

[7] E. D. Sontag, "Monotone and near-monotone biochemical networks." Syst Synth Biol, vol. 1, no. 2, pp. 59-87, 2007.

[8] P. de Leenheer, D. Angeli, and E. D. Sontag, "Monotone chemical reaction networks," Journal of Mathematical Chemistry, vol. 41, no. 3 , pp. 295-314, 2007.

[9] C. D. Thron, "The secant condition for instability in biochemical feedback control - Parts I and II." Bulletin of Mathematical Biology, vol. 53, pp. 383-424, 1991.

[10] J. J. Tyson and H. G. Othmer, "The dynamics of feedback control circuits in biochemical pathways." in Progress in Theoretical Biology, R. Rosen and F. M. Snell, Eds. Academic Press, 1978, vol. 5, pp. $1-62$.

[11] M. Arcak and E. D. Sontag, "Diagonal stability of a class of cyclic systems and its connection with the secant criterion," Automatica, vol. 42, no. 9, pp. 1531-1537, 2006

[12] _ _ "A passivity-based stability criterion for a class of biochemical reaction networks," Mathematical Biosciences and Engineering, vol. 5, no. 1, pp. 1-19, 2008.

[13] F. A. Chandra, G. Buzi, and J. C. Doyle, "Linear control analysis of the autocatalytic glycolysis system," in Proc American Control Conference, 2009, pp. 319-324.

[14] J. Coatleven and C. Altafini, "A kinetic mechanism inducing oscillations in simple chemical reactions networks," in Proc Conf on Decision and Control, 2008, pp. 1771-1776.

[15] M. Banuelos, C. Gancedo, and J. M. Gancedo, "Activation by phosphate of yeast phosphofructokinase." Journal of Biological Chemistry, vol. 252, no. 18, pp. 6394-6398, 1977.

[16] S. Dano, M. F. Madsen, H. Schmidt, and G. Cedersund, "Reduction of a biochemical model with preservation of its basic dynamic properties," FEBS Journal, vol. 273, no. 21, 2006.

[17] J. Ovadi and V. Saks, "On the origin of intracellular compartmentation and organized metabolic systems," MOLECULAR AND CELLULAR BIOCHEMISTRY, vol. 256, no. 1-2, pp. 5-12, JAN-FEB 2004. 